\title{
REGULATION OF P53 BY SIRNA IN RADIATION TREATED CELLS: SIMULATION STUDIES
}

\author{
KRZYSZTOF PUSZYŃSKI， ROMAN JAKSIK，ANDRZEJ ŚWIERNIAK
}

Institute of Automatic Control

Silesian University of Technology, Akademicka 16, 44-100 Gliwice, Poland e-mail: \{krzysztof.puszynski,roman.jaksik, andrzej.swierniak\}@polsl.pl

\begin{abstract}
Ionizing radiation activates a large variety of intracellular mechanisms responsible for maintaining appropriate cell functionality or activation of apoptosis which eliminates damaged cells from the population. The mechanism of such induced cellular death is widely used in radiotherapy in order to eliminate cancer cells, although in some cases it is highly limited by increased cellular radio-resistance due to aberrations in molecular regulation mechanisms of malignant cells. Despite the positive correlation between the radiation dose and the number of apoptotic cancer cells, radiation has to be limited because of extensive side effects. Therefore, additional control signals whose role will be to maximize the cancer cells death-ratio while minimizing the radiation dose and by that the potential side effects are worth considering. In this work we present the results of simulation studies showing possibilities of single gene regulation by small interfering RNA (siRNA) that can increase radio-sensitivity of malignant cells showing aberrations in the p53 signaling pathway, responsible for DNA damage-dependant apoptosis. By blocking the production of the p53 inhibitor Mdm2, radiation treated cancer cells are pushed into the apoptotic state on a level normally achievable only with high radiation doses. The presented approach, based on a simulation study originating from experimentally validated regulatory events, concerns one of the basic problems of radiotherapy dosage limitations, which, as will be shown, can be partially avoided by using the appropriate siRNA based control mechanism.
\end{abstract}

Keywords: siRNA, p53, combined therapy.

\section{Introduction}

Ionizing radiation is one of the most widely used factors in cancer treatment due to its ability to activate programmed cellular death, called apoptosis, in a specific targeted population of cancer cells. Its efficiency depends significantly on the radiation dose used, although its limit is set by very severe side effects, which may lead to additional outbreaks of cancer and premature death. Radiation affects a majority of the intracellular microelements, out of which the most critical ones include the chromosomes, which contain genetic material in the form of a double stranded DNA helix.

Ionizing radiation induces a large variety of damages in the DNA including Double Strand Breaks (DSBs). DNA integrity is constantly monitored by a variety of molecular mechanisms controlled mainly by the p53 protein (Kohn and Pommier, 2005; Levine et al., 2006). The role of p53 is to protect the DNA by activating a specific signaling cascade built of hundreds of genes and their protein products. Concentration of p53 depends on the scale of the DNA damage, allowing preventing their harmful effects by activation of three very important intracellular mechanisms. High oscillation peaks of the p53 levels lead first to cell cycle arrest preventing the cell from copying the damaged DNA to the daughter cells, and then it activates a large variety of DNA repair mechanisms. Finally, in the case of their inefficiency caused mainly by high extensiveness of the damage, p53's final role after reaching high level of concentration is to trigger apoptosis, which eliminates the defective cell from the population.

In normal conditions the p53 level is typically low under the control of the $\mathrm{Mdm} 2$ protein responsible for its degradation (Haupt et al., 1997). Synthesis of Mdm2 depends on p53 itself, creating a negative feedback loop which is very commonly observed in intracellular regulation mechanisms. Another crucial element of the p53 signaling cascade is a positive feedback involving PTEN, PIP3 and Akt proteins, promoting p53 production under certain stress conditions (Harris and Levine, 2005). 
The existence of the negative feedback assures homeostasis of healthy cells and oscillatory responses of DNA-damaged cells, which are persistent when DNA repair is inefficient and the positive feedback loop is broken (like in MCF-7 cancer cells). The positive feedback destroys the negative coupling between Mdm2 and $\mathrm{p} 53$ by sequestering most of Mdm2 in the cytoplasm, so it may no longer promote the nuclear p53 for degradation. This positive feedback loop works as a countdown clock: it gives the cell some time for DNA repair, but when the repair is inefficient, it increases the level of active p53 and triggers transcription of proapoptotic genes. As a result, small DNA damage may be repaired and the cell may return to its initial "healthy" state, while extended damage results in apoptosis.

Mutations or deletions in the DNA sequence that occur during tumor development may deactivate certain elements of the p53 pathway. One of the most often deactivated proteins in malignant cells is PTEN, which due to structural damages may lose its enzymatic activity, leading to increased cell proliferation and reduced cell death, resulting in a rapid tumor development (reviewed by Li and Ross (2007)). Inactivation of PTEN may be observed among many cancer cell lines, like MCF-7, which show very high resistance to radiotherapy due to an impaired ability to trigger apoptosis (Geva-Zatorsky et al., 2006).

Inactivation of a certain regulatory element due to DNA mutations or deletions presents a big challenge for the processes which aim to restore appropriate regulation, since in most cases their natural role cannot be restored despite large possibilities of their concentration controlling mechanisms. In such situations the only solution may be to control an additional element of the regulation system in order to achieve a similar effect.

In this work we present the possibilities of a targeted Mdm2 regulation, based on siRNA (small interfering RNA) particles that allows omitting PTEN deficiency and trigger apoptosis in irradiated cancer cells. For this purpose we use our previous deterministic p53 signaling pathway model (Puszynski et al., 2008) expanded by an additional siRNA based Mdm2 regulation, incorporating a process called RNA interference (RNAi). The ability to increase cells radio-sensitivity by a targeted, gene specific therapy may provide new insights into radiotherapy methods reducing high dosage use and therefore limiting the side effects.

The possibilities of a targeted, siRNA based, single gene regulation in cancer treatment were shown by Davis (2009), allowing to target only cancer cell subpopulation by the use of polymer based nanoparticle carriers. In this work we focus on the ability to control siRNA based Mdm2 transcript degradation efficiency and its consequences, omitting the process of siRNA transport into cancer cells, which corresponds to in vitro conditions.
In such a case, high siRNA efficiency is much easier to achieve and does not require cell-specific targeting mechanisms. Additionally, the effects of regulated Mdm2 deficiency on cellular radio-sensitivity are simulated based on models representing normal and cancer, PTEN deficient cells using existing the MCF-7 cancer cell line as a reference.

The RNA interference mechanism introduced in the presented model is a highly conserved regulatory system based on a sequence-specific degradation of mRNA molecules. RNAi incorporates small RNA particles which, based on a specific nucleotide sequence motif, recognize its target mRNAs, resulting in translational silencing or transcript degradation.

The most widely used regulatory agent of RNAi is a double stranded siRNA molecule, about 22 nt long, which can be introduced directly into the cells in its mature form or as a long double stranded (dsRNA) or a short hairpin (shRNA) precursor, allowing us to specifically control the degradation level of complementary mRNA molecules. siRNAs transfected into the cells become a part of a multiprotein complex called RISC, responsible for their processing and target transcript cleavage, leading to its degradation ( $\mathrm{Li}$ and $\mathrm{Cha}$, 2007). After successful interference the complex can continue to its next target, recognized on the basis of a unique siRNA nucleotide sequence, repeating the process until it loses its internal stability. The basic assumption of siRNA based gene silencing is that it functions independently of the natural cell development system and is gene-specific, which in many cases cannot be achieved due to various experiment-related factors.

Since its discovery (Fire et al., 1998), the siRNA potential was harnessed in many areas of biomedical research becoming a very popular molecular biology tool used for gene function analysis as well as a therapeutic agent permitting to control specific elements of a pathogenic regulatory pathway. The ability to control single elements of an intracellular regulation system by gene expression silencing or production of knock-out phenotypes brings new insights into the development of therapeutics against cancer, viral infections and autoimmune disorders (Ryther et al., 2005; Hannon and Rossi, 2004; Dorsett and Tuschl, 2009) unattainable with the standard medicines.

The main concerns of siRNA treatment which must be addressed during model creation include the dosing schedule, the dynamics of the target gene expression and the efficiency of siRNA transport into the cells. Previous studies have shown that naked siRNA can work effectively after being transfected into the cells using either local or systematic delivery (Kim et al., 2004). The usage of transporting agents such as polymer based nanoparticles (Davis, 2009), plasmid or viral vectors can significantly enhance siRNA efficiency, additionally allowing targeting 
specific cell types (Shim and Kwon, 2010; Makinen et al., 2006).

The duration of the siRNA-induced gene silencing effect lasts from a few hours to a few months, depending mainly on the transporting method and chemical stabilisation, limited by the siRNA and transporting agent toxicity level. Moreover, a small siRNA dose may lead only to a partial silencing effect in a very short period of time. In contrast, an excessive dose may trigger an interferon response, increasing the siRNA degradation speed, leading to decreased effectiveness and also in many cases being lethal to the organism. An appropriate dosing schedule and transporting mechanisms, combined with high specificity of the siRNA sequence, are therefore the biggest challenges for the efficient use of siRNA.

In the presented model, siRNA is used to control the concentration of the Mdm2 protein level by increasing the MDM2 transcript degradation rate, which, as previously shown, can be achieved with high efficiency using siRNAs of a specific sequence (Giono and Manfredi, 2007). A decreased concentration of $\mathrm{Mdm} 2$ increases the level of p53 protein responsible for DNA damage induced apoptosis. Under such conditions the apoptotic pathway can be activated with a significantly smaller amount of double strand DNA breaks, which means that a much lower dose of ionizing radiation is required to activate the signaling cascade leading to cellular death.

\section{Computational model of siRNA based regulation}

Mathematical modelling as a tool for cancer therapy has a long history (see, e.g., the work of Świerniak et al. (2003) and the references therein). In our previous work we proposed a deterministic and stochastic model of p53 regulation in response to DNA damage (Puszynski et al., 2008), which is based on two feedback loops: the negative, coupling p53 with its immediate downregulator Mdm2, and the positive, which involves PTEN, PIP3 and Akt (Fig. 1). In such a representation the time evolution of the system is equivalent to a piece-wise deterministic, time-continuous Markov process.

Due to a very well described apoptosis blockage mechanism, MCF-7 cells are considered in the model as an example of the cancer cells. They are known for a mutated and therefore inactive PTEN gene (García et al., 2004; Krawczyk et al., 2007), which is represented in the model by setting the number of PTEN gene copies to zero. Our previous deterministic p53 interaction model consists of 13 nonlinear differential equations describing time evolution of relevant molecules concentrations (Puszynski et al., 2008, Eqns. (8)-(15), (19), (21), (23)), with two modified equations for Mdm2 and PTEN genes (cf. Eqn. (5) therein). It was extended by the siRNA based regulation, including its dynamics and efficiency, originating from publicly available experimental studies. Model modifications include a new equation describing the change in the intracellular siRNA amounts:

$$
\frac{\mathrm{d}}{\mathrm{d} t} \operatorname{siRNA}=p_{2} \cdot i R N A-d_{12} \cdot \operatorname{siRNA},
$$

where iRNA is the extracellular siRNA concentration, and modification of the equation describing the change ratio of the Mdm2 mRNA by adding the last term:

$$
\begin{aligned}
\frac{\mathrm{d}}{\mathrm{d} t} M d m \mathscr{2}_{t}= & s_{0} \cdot G m d m \mathscr{2}-d_{7} \cdot M d m \mathscr{2}_{t} \\
& -d_{11} \cdot M d m \mathscr{2}_{t} \cdot \frac{\operatorname{siRNA}}{\operatorname{siRNA+h_{1}}} .
\end{aligned}
$$

The Michaelis-Menten form of this term provides some saturation on the level of siRNA causing mRNA degradation, so there is no possibility of $100 \%$ efficiency of this reaction, which is consistent with experimental data shown in the literature.

The most critical elements of siRNA regulation that need to be addressed in the model include siRNA lifetime $d_{12}$, which without any chemical stabilization lasts a couple of minutes (about 8 in the model) and the siRNA diffusion rate into the cells $p_{2}$. Delivery of siRNA into the cell is not a trivial problem. As show by Veldhoen et al. (2006), intracellular siRNA concentration is typically lower than $10 \%$ of the extracellular one, depending on the transporting medium and inhibitors/effectors used. In our model we set $p_{2}$ to receive $8 \%$, which is generally achievable in the experiments. The siRNA uptake percentage is independent of its concentration at least when considering the doses in a range of 0.1 to 100 $\mathrm{nM}$, where the relationship between the extracellular and intracellular siRNA level was shown to be linear (Veldhoen et al., 2006; Overhoff et al., 2004). This justifies approximation of the input term in Eqn. (1).

An mRNA degradation by siRNA interactions is another very important element. Based on the information provided by commercial siRNA manufacturers, the model parameters $d_{11}$ and $h_{1}$ are set so that 30nM siRNA concentration outside of the cell allows to achieving $75 \%$ silencing efficiency. siRNA dosage described in the literature usually exceeds the suggested $30 \mathrm{nM}$, reaching 100 to $300 \mathrm{nM}$. In order to additionally validate if such an extensive dose does not trigger apoptosis in normal cells, we have also checked the model response at a dose of 300 nM siRNA.

\section{Simulation results}

All results are obtained with parameter values shown in Table 1 or taken as in the base model (Puszynski et al., 2001, Table 1).

Under normal conditions, untreated cells show no DNA damages (represented by double strand breaks) and 


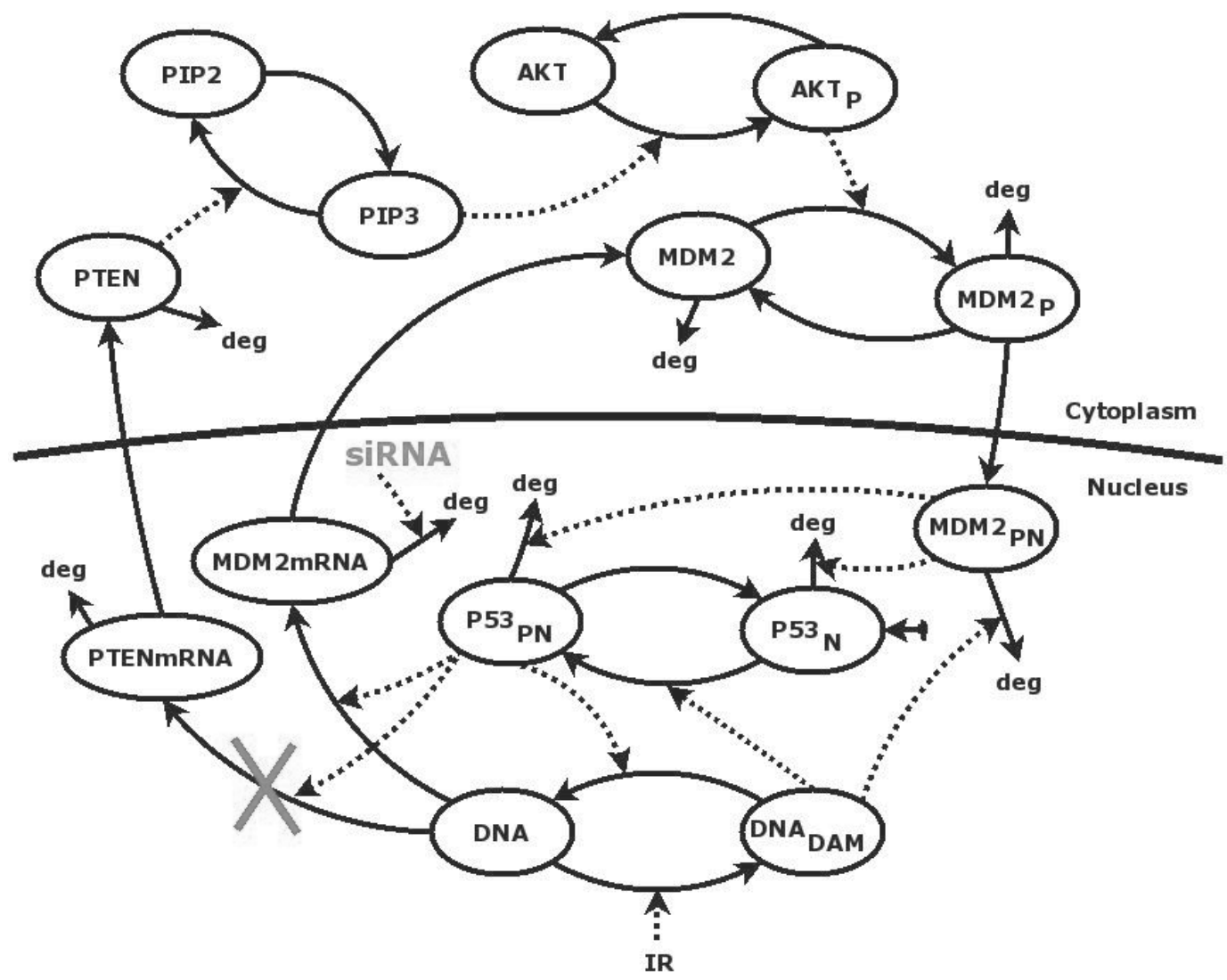

Fig. 1. Model of the p53|Mdm2 signaling pathway with PTEN production blockade (MCF-7 cell line) and Mdm2 degradation regulated by proper siRNA.

Table 1. New parameters.

\begin{tabular}{|l|l|l|}
\hline Name & Meaning & Value \\
\hline \hline$p_{2}$ & siRNA import to cell rate & 0.2 \\
$s_{0}$ & Mdm2 mRNA transcription rate & 0.06 \\
$h_{1}$ & M-M coefficient & 8600 \\
$d_{7}$ & Mdm2 mRNA standard degradation rate & $3 \cdot 10^{-4}$ \\
$d_{11}$ & Mdm2 mRNA caused by siRNA degradation rate & $3.5 \cdot 10^{-3}$ \\
$d_{12}$ & siRNA degradation rate & $2 \cdot 10^{-3}$ \\
\hline
\end{tabular}

the siRNA concentration levels are equal to zero (Fig. 2(a)). p53 is maintained at a very low level due to its inhibitor Mdm2, which shows high concentration in a system with a properly functioning negative Mdm2 feedback loop.

Figure 2(b) shows that even a 24-hour pulse of 300 nM Mdm2-specific siRNA does not generate any severe regulatory disorders. After a short-term Mdm2 level drop and resulting p53 increase, by a value insignificant for the proper functioning of the cell, the p53 level returns to its initial state as soon as the siRNA level drops.

Healthy cells subjected to a small radiation dose of 1 Gy experience DNA damages that result in p53-Mdm2 oscillations. Such a dose generates not enough DNA damages in order to activate apoptosis, since they are efficiently dealt with by the intracellular DNA repair systems. The positive feedback loop, which works as a timer in the presence of DNA damages, has no enough time for activation and the cell finally returns to its initial state (Fig. 2(c)).

As shown by Puszynski et al. (2008), the base model has a bifurcation point at $1.88 \mathrm{~Gy}$, so the increase in the radiation dose to 2 Gy changes the response of healthy cells. Due to insufficient performance of the repair systems the increased amount of DNA damages cannot be removed before the activation of the positive feedback loop. As a result, the Mdm2 activation and its cell nucleus entry are suspended. p53 reaches a high level, which leads to the initiation of apoptosis. In this state the cell activates a wide range of enzymes including caspases that indirectly lead to DNA shattering by exponentially raising the level of DSB, which eventually leads to cellular death. The 
(a) Normal cells without IR or siRNA stimulation

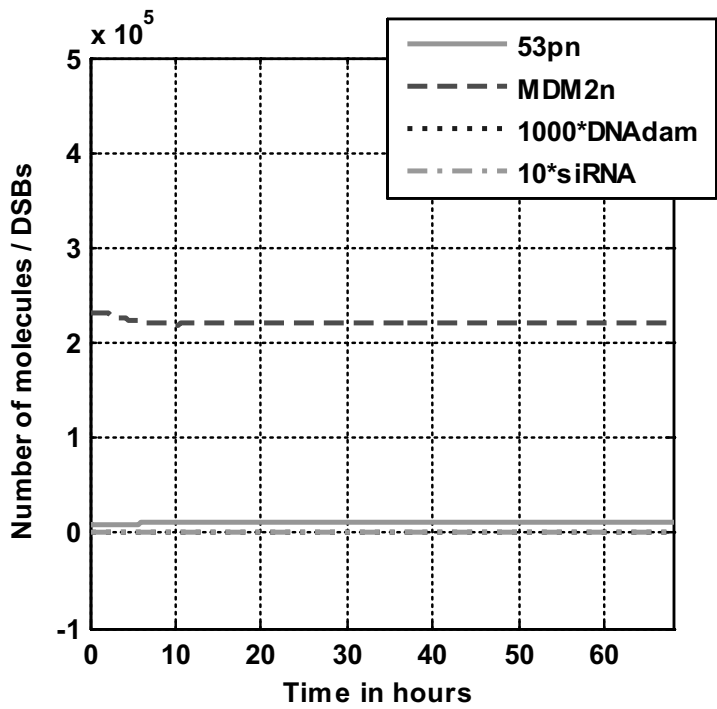

(c) Normal cells with IR stimulation (1 Gy)

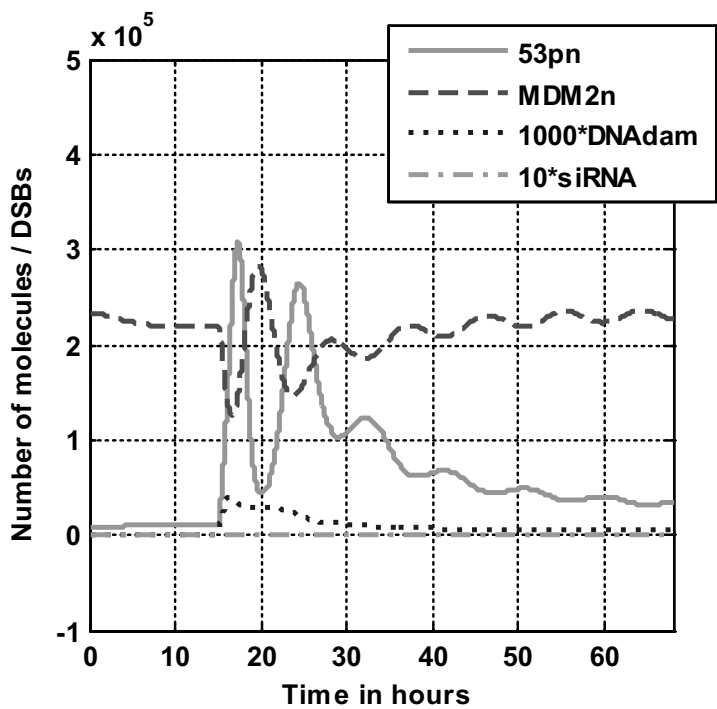

(b) Normal cells with 24h siRNA stimulation (300 nM)

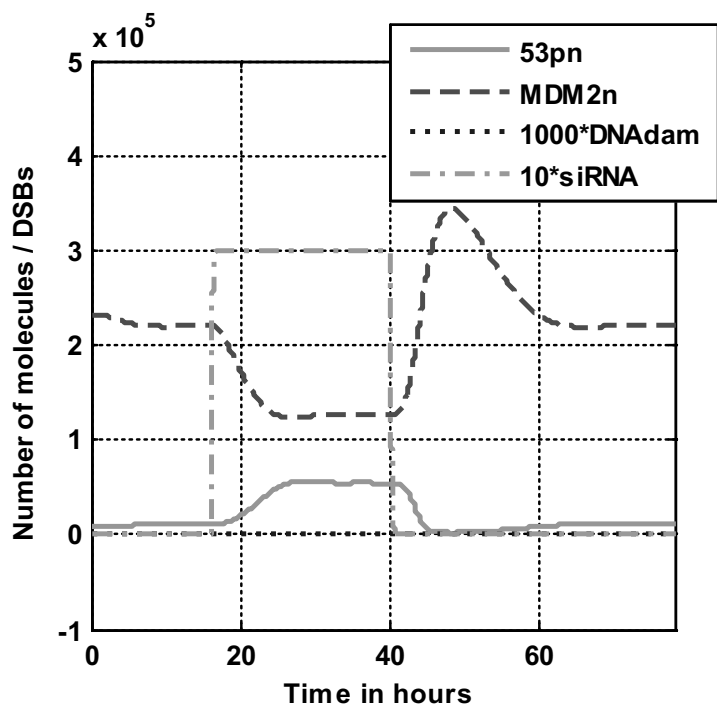

(d) Normal cells with IR stimulation (2 Gy)

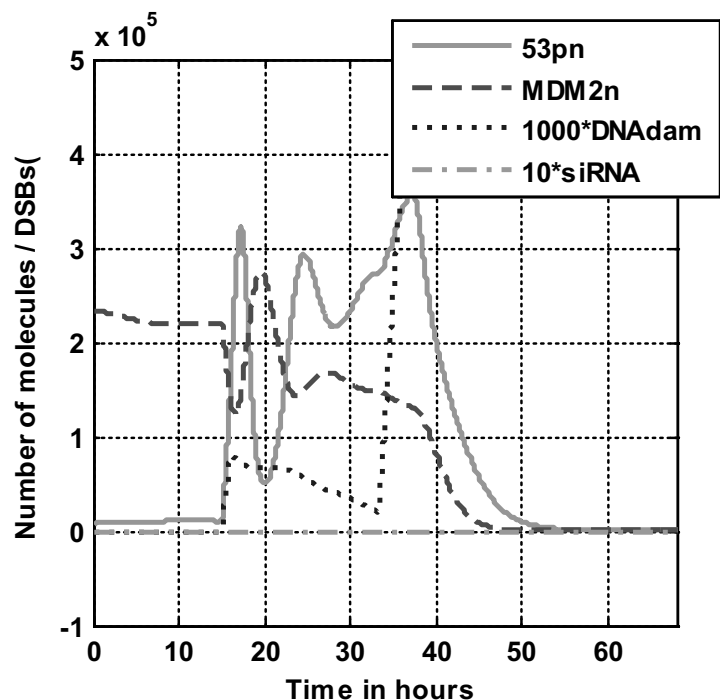

Fig. 2. Normal cells behavior without any stimulation (a), after $24 \mathrm{~h}$ stimulation of siRNA at a dose of $300 \mathrm{nM}$ (b), after IR at a dose of $1 \mathrm{~Gy}$-survival (c) and $2 \mathrm{~Gy}$-apoptosis (d).

rapidly increasing amount of DNA damages blocks the production of new proteins, which combined with their direct caspase-dependent cleavage, leads to their overall decrease in the cell (Fig. 2(d)).

Due to the blocked positive feedback loop and despite inefficient DNA repair mechanisms, in the MCF-7 cells the apoptosis state is not initialized (Fig. 3(a)). MCF-7 cells subjected to radiation show prolonged oscillations in the levels of p53 and Mdm2 as shown by Geva-Zatorsky et al. (2006).

MCF-7 cells were treated with a minimal dose of $30 \mathrm{nM}$ siRNA, which allows achieving at least $75 \%$ of silencing of the Mdm2 gene for a period of 2 hours. Even such a short, low dosage transfection resulted in an effect similar to the one obtained in a properly functioning positive feedback loop, where the Mdm2 level drops significantly activating p53. In such conditions MCF-7 cells act as cells with an undamaged p53 pathway and enter the apoptotic state after 2 Gy of irradiation (Fig. 3(b)).

Increasing the siRNA dose to $100 \mathrm{nM}$ ( $2 \mathrm{~h}$ pulse $)$ allows further decreasing radiation dose necessary to initiate apoptosis to $0.5 \mathrm{~Gy}$ (Fig. 3(c)). Both factors, 0.5 Gy radiation and $100 \mathrm{nM}$ siRNA, working separately are not lethal to the healthy cells since at least $2 \mathrm{~Gy}$ is required to initiate apoptosis and, as shown in Fig. 2(b), even $300 \mathrm{nM}$ of siRNA does not induce significant changes in the p53 signaling pathway that would be harmful to the cell. The combined power of two "weak" control signals is however enough to push cancer MCF-7 cells 
(a) MCF-7 cells with IR stimulation (2 Gy)

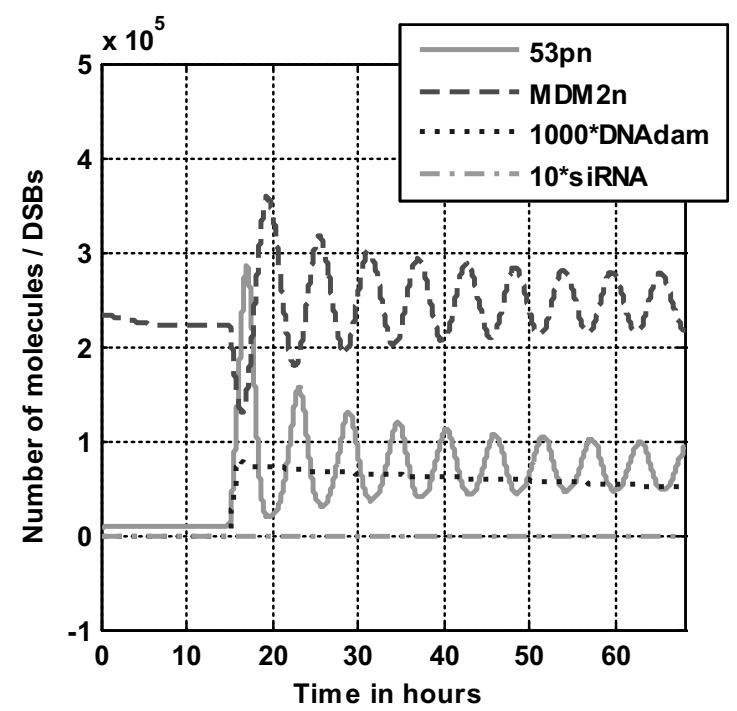

(c) MCF-7 cells with IR (0.5 Gy) and $2 \mathrm{~h}$ siRNA (100 nM)

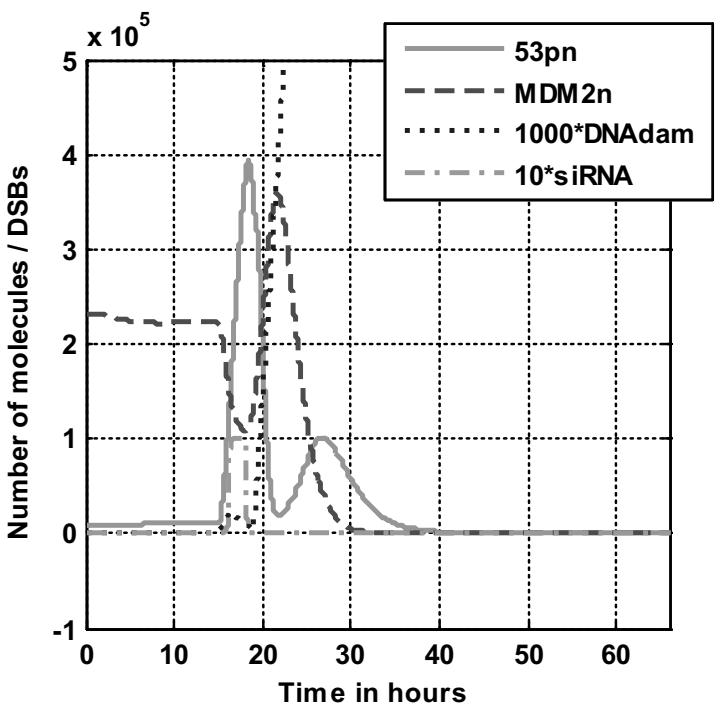

(b) MCF-7 cells with IR ( 2 Gy) and $2 \mathrm{~h}$ siRNA ( $30 \mathrm{nM})$

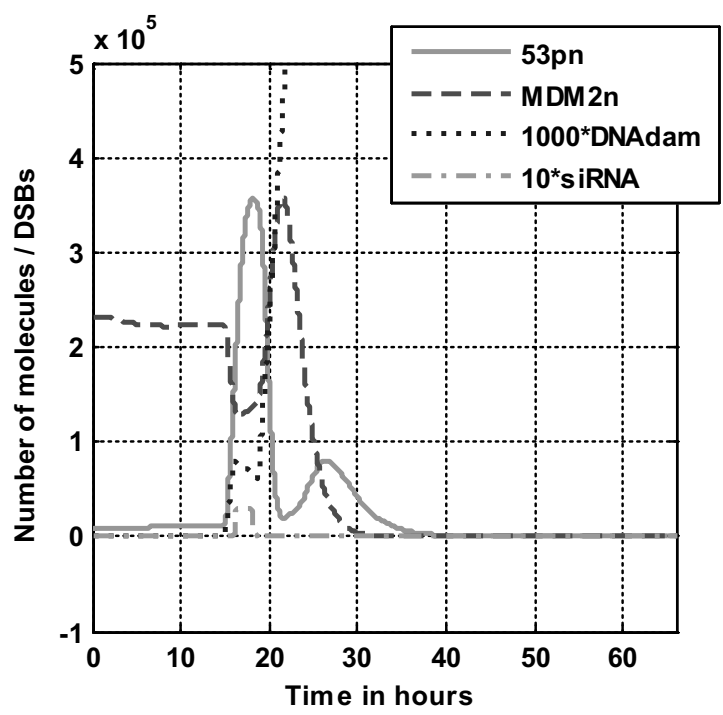

(d) Minimal siRNA dose vs exposure time required to

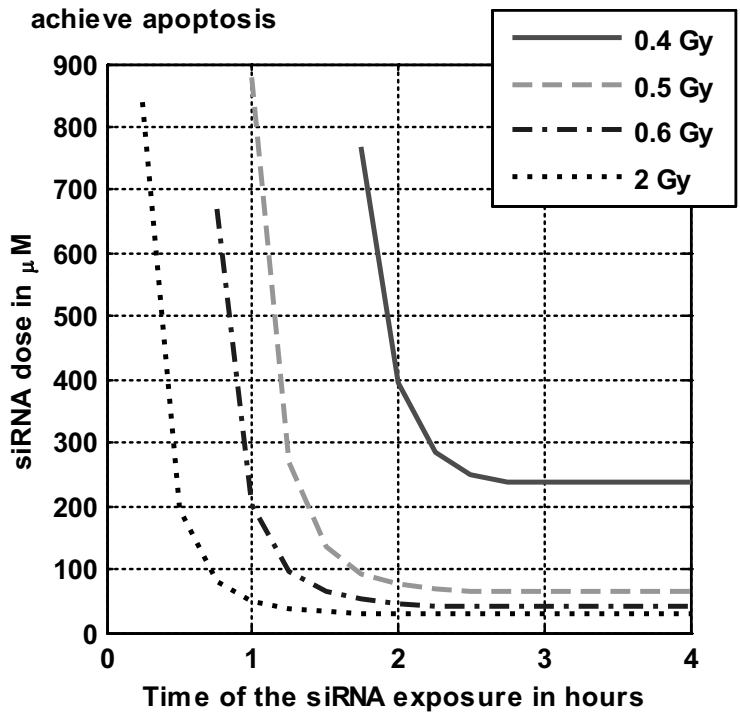

Fig. 3. MCF-7 cells behavior after IR radiation at a dose of 2 Gy-survival (a), after IR radiation at a dose of 2 Gy followed by $2 \mathrm{~h}$ siRNA transfection at a dose of $30 \mathrm{nM}$-apoptosis (b) and after IR radiation at a dose of 0.5 Gy followed by a $2 \mathrm{~h}$ siRNA transfection at a dose of $100 \mathrm{nM}$-apoptosis (c), minimal siRNA dose vs. time exposure required to achieve apoptosis in MCF-7 cells (d).

to death. Figure 3(d) presents relationship between the minimal siRNA dose and time exposure required to achieve apoptosis in MCF-7 cells depending on the irradiation dose.

\section{Concluding remarks}

The study presents the concept of using siRNA as a supporting control for the main regulation based on ionizing radiation. The role of supporting control is to increase cellular susceptibility for the main controlling factor, in particular, to allow the cells with damaged positive feedback loop to enter the apoptotic pathway.
The increased radiation sensitivity allows significant reduction in its dose and, consequently, avoiding extensive side effects, which are one of the basic problems in radiotherapy. The technical possibilities of a siRNA delivery system which allows targeting only cancer cells, like for example, that presented by Gatter et al. (1983), permit to increase radiation sensitivity without inducing significant changes among healthy cells. This may provide a basis for the development of new targeted therapy protocols in which cancer cells subjected to much lower radiation doses undergo apoptosis and at the same time the reduced level of radiation does not cause the death of healthy cells. 
The administration protocol used in the simulations concerning $2 \mathrm{~h}$ siRNA pulse with a dose of $30 \mathrm{nM}$ (Fig. 3(b)) involves the minimum dose and exposure time adjusted for the 2 Gy irradiation, required to achieve the apoptosis effect in the MCF-7 cells (Fig. 3(d)). A longer exposure time or a higher siRNA dose do not cause cells to change their behavior and under a dose of 2 Gy of radiation they still continue to undergo apoptosis. Reduction in the radiation dose results in the need to increase the dose of siRNA and/or the exposure time. Even with the lowest radiation dose necessary to achieve apoptosis $(0.4 \mathrm{~Gy})$, we still are able to find a siRNA dose which when combined with the 2 hour exposure time, leads to the death of MCF-7 cells. This is important considering the relatively short siRNA half-life and the trouble associated with its delivery into a specific location of the organism.

Even if it seems that a large dose of siRNA could completely replace the radiation, such an event does not appear possible. The reason is a limited ability of the cells to absorb siRNA from the environment as well as a competition between the rate of mRNA degradation and its translation. Despite high levels of siRNA it is impossible to achieve $100 \%$ silencing efficiency and thus silence the gene completely, especially in in vivo conditions. Attainable levels of the silencing range between 75 and $90 \%$ with $75 \%$ being the guaranteed minimum by most of the siRNA manufacturers. For this reason siRNA remains only a supporting control requiring radiation for the initiation of apoptosis.

The proposed concept of using two weak control signals in order to achieve apoptosis instead of one strong signal may be expanded into other types of controlling factors like UV radiation, Nutlin-3, miRNA or TNF. The specificity of siRNA based regulation also allows targeting additional signaling pathway elements, in cells with deficiencies other than PTEN inactivation. The p53 pathway may be impaired by various mutations in the TP53 gene or other crucial pathway elements like AKT or MDM2 (Hrstka et al., 2009; Goldstein et al., 2011), resulting in an increased survivability of malignant cells. The main challenge related to the proposed approach is to identify the points in which anticancer signaling pathways are broken along with their possible bypasses. This search may be aided by systems biology and its comprehensive description of regulatory events. The possibility of bypassing impaired regulatory systems in order to achieve the appropriate cell response might lead to the development of new, less harmful, targeted and personalized therapies with much less noticeable side-effects. Despite the encouraging simulation results, the proposed approach requires complex experimental verification both in vivo and in vitro in order to confirm its effectiveness as a new approach to anti-cancer treatment in PTEN deficient cells. During these experiments the conclusions of Fujarewicz (2010) about the design of identification experiments should be taken into account.

\section{Acknowledgment}

The authors would like to thank the anonymous reviewers for their comments and suggestions, which helped to improve the manuscript.

This work was supported by the National Science Centre (NCN) grants no. N N519 647840 (for Andrzej Świerniak and Roman Jaksik) and N N518 287540 (for Krzysztof Puszyński).

\section{References}

Davis, M.E. (2009). The first targeted delivery of siRNA in humans via a self-assembling, cyclodextrin polymer-based nanoparticle: From concept to clinic, Molecular Pharmacology 6(3): 659-668.

Dorsett, Y. and Tuschl, T. (2009). siRNAs: Applications in functional genomics and potential as therapeutics, Nature Reviews Drug Discovery 3(4): 318-329.

Fire, A., Xu, S., Montgomery, M., Kostas, S., Driver, S. and Mello, C. (1998). Potent and specific genetic interference by double-stranded RNA in Caenorhabditis elegans, $\mathrm{Na}$ ture 391(6669): 806-811.

Fujarewicz, K. (2010). Planning identification experiments for cell signaling pathways: An $\mathrm{NF} \kappa \mathrm{B}$ case study, International Journal of Applied Mathematics and Computer Science 20(4): 773-780, DOI: 10.2478/v10006-010-0059-6.

García, J., Silva, J., Peña, C., Garcia, V., Rodríguez, R., Cruz, M.A., Cantos, B., Provencio, M., España, P. and Bonilla, F. (2004). Promoter methylation of the PTEN gene is a common molecular change in breast cancer, Genes Chromosomes and Cancer 41(2): 117-124.

Gatter, K., Brown, G., Trowbridge, I., Woolston, R. and Mason, D. (1983). Transferring receptors in human tissues: Their distribution and possible clinical relevance, Journal of Clinical Pathology 36(5): 539-545.

Geva-Zatorsky, N., Rosenfeld, N., Itzkovitz, S., Milo, R., Sigal, A., Dekel, E., Yarnitzky, T., Liron, Y., Polak, P., Lahav, G. and Alon, U. (2006). Oscillations and variability in the p53 system, Molecular Systems Biology 2: 2006.0033

Giono, L. and Manfredi, J. (2007). Mdm2 is required for inhibition of cdk2 activity by $\mathrm{p} 21$, thereby contributing to p53-dependent cell cycle arrest, Molecular Cell Biology 27(11): 4166-4178.

Goldstein, I., Marcel, V., Olivier, M., Oren, M., Rotter, V. and Hainaut, P. (2011). Understanding wild-type and mutant p53 activities in human cancer: New landmarks on the way to targeted therapies, Cancer Gene Therapy 18(1): 2-11.

Hannon, G. and Rossi, J. (2004). Unlocking the potential of the human genome with RNA interference, Nature 431(7006): 371-378.

Harris, S. and Levine, A. (2005). The p53 pathway: Positive and negative feedback loops, Oncogene 24(17): 2899-2908. 
Haupt, Y., Maya, R., Kazaz, A. and Oren, M. (1997). Mdm2 promotes the rapid degradation of p53, Nature 387(6630): 296-299.

Hrstka, R., Coates, P. and Vojtesek, B. (2009). Polymorphisms in p53 and the p53 pathway: Roles in cancer susceptibility and response to treatment, Journal of Cellular and Molecular Medicine 13(3): 440-453.

Kim, B., Tang, Q., Biswas, P., Xu, J., Schiffelers, R., Xie, F., Ansari, A., Scaria, P., Woodle, M., Lu, P. and Rouse, B. (2004). Inhibition of ocular angiogenesis by siRNA targeting vascular endothelial growth factor pathway genes: Therapeutic strategy for herpetic stromal keratitis, The American Journal of Pathology 165(6): 2177-2185.

Kohn, K. and Pommier, Y. (2005). Molecular interaction map of the p53 and mdm2 logic elements, which control the off-on switch of p53 in response to DNA damage, Biochemical and Biophysical Research Communications 331(3): 816-827.

Krawczyk, B., Rudnicka, K. and Fabianowska-Majewska, K. (2007). The effects of nucleoside analogues on promoter methylation of selected tumor suppressor genes in mcf-7 and mda-mb-231 breast cancer cell lines, Nucleosides, $\mathrm{Nu}$ cleotides and Nucleic Acids 26(8-9): 1043-1046.

Levine, A., Hu, W. and Feng, Z. (2006). The p53 pathway: What questions remain to be explored?, Cell Death and Differentiation 13(6): 1027-1036.

Li, L. and Ross, A. (2007). Why is PTEN an important tumor suppressor?, Journal of Cellular Biochemistry 102(6): 1368-1374.

Li, W. and Cha, L. (2007). Predicting siRNA efficiency, Cellular and Molecular Life Sciences 64(14): 1785-11792.

Makinen, P., Koponen, J., Karkkainen, A., Malm, T., Pulkkinen, K., Koistinaho, J., Turunen, M. and Yla-Herttuala, S. (2006). Stable RNA interference: Comparison of u6 and $\mathrm{h} 1$ promoters in endothelial cells and in mouse brain, The Journal of Gene Medicine 8(4): 433-441.

Overhoff, M., Wnsche, W. and Sczakiel, G. (2004). Quantitative detection of siRNA and single-stranded oligonucleotides: Relationship between uptake and biological activity of siRNA, Nucleic Acids Research 32(21): e170.

Puszynski, K., Hat, B. and Lipniacki, T. (2008). Oscillations and bistability in the stochastic model of p53 regulation, Journal of Theoretical Biology 254(2): 452-465.

Ryther, R., Flynt, A., Phillips, J. and Patton, J. (2005). siRNA therapeutics: Big potential from small RNAs, Gene Therapy 12(1): 5-11.
Shim, M. and Kwon, Y. (2010). Efficient and targeted delivery of siRNA in vivo, FEBS Journal 277(23): 4814-4827.

Świerniak, A., Ledzewicz, U. and Schättler, H. (2003). Optimal control for a class of compartmental models in cancer chemotherapy, International Journal of Applied Mathematics and Computer Since 13(3): 357-368.

Veldhoen, S., Laufer, S.D., Trampe, A. and Restle, T. (2006). Cellular delivery of small interfering RNA by a non-covalently attached cell-penetrating peptide: Quantitative analysis of uptake and biological effect, $\mathrm{Nu}$ cleic Acids Research 34(22): 6561-6573.

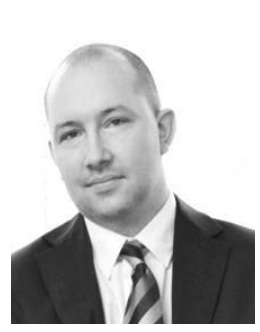

Krzysztof Puszyński received his M.Sc. degree in automatic control and robotics from the Silesian University of Technology in 2003 and a Ph.D. in biocybernetics and biomedical engineering from the same university in 2009. His main research interests include systems biology, bioinformatics and control engineering in biological systems. He was a visiting professor at the Institute of Systems Analysis and Informatics in Rome in 2011 and at the University of Alberta in Edmonton, Canada, in 2012. He is presently an assistant professor at the Department of Automatic Control, Silesian University of Technology. He is the author or co-author of more than 35 journal articles, book chapters and conference papers.

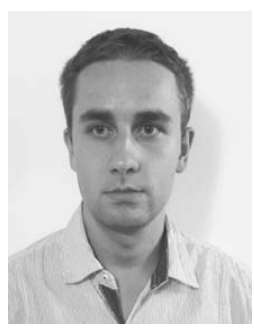

Roman Jaksik received his M.Sc. degree in automatic control and robotics from the Silesian University of Technology (Gliwice, Poland) in 2008, where he continues his education as a Ph.D. student. His research interests include systems biology, genetic engineering and bioinformatics.

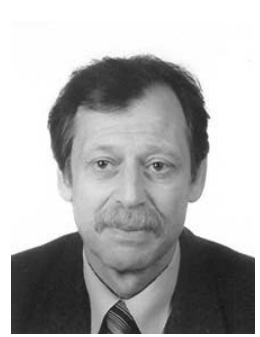

Andrzej Świerniak received his Ph.D. and D.Sc (habilitation) degrees in control engineering from the Silesian University of Technology, Gliwice, Poland, in 1978 and 1988, respectively. $\mathrm{He}$ is presently a full professor of automatic control and bioinformatics, and the head of the Department of Automatic Control, Silesian University of Technology. Professor Świerniak is an author and coauthor of more than 250 journal articles, book chapters and conference papers, a guesteditor of special issues of Mathematical Biosciences and Engineering, Archives of Control Sciences, the International Journal of Applied Mathematics and Computer Science, and an advisor of the Journal of Biological Systems. His main research interests include the theory of optimal and robust control and its applications in molecular biology, biotechnology, bioinformatics and medicine. 